\title{
Nichts ist beständiger als der Wandel - Neues Editorenteam bei der Zeitschrift Grundwasser
}

\author{
Gudrun Massmann • Traugott Scheytt
}

Online veröffentlicht: 05.02.2014

(C) Springer-Verlag Berlin Heidelberg 2013

Liebe Leserinnen und Leser,

mit großem Engagement haben Johannes Barth und Rudi Liedl zusammen mit den assoziierten Editoren die Zeitschrift Grundwasser in den vergangenen vier Jahren gestaltet. Mit Ihrem Einsatz haben sie, wie auch die vorangegangenen Editorenteams, gemeinsam mit den Autoren der Beiträge dafür gesorgt, dass sich Grundwasser als Sprachrohr der Hydrogeologen und Wasserwirtschaftler im deutschsprachigen Raum etabliert hat. Fast 200 Billionen Liter Grundwasserneubildung später verabschieden sich Johannes Barth und Rudi Liedl nun in ihren verdienten Editoren-,,Ruhestand“. An dieser Stelle sei Ihnen und den assoziierten Editoren der vergangenen vier Jahre noch einmal ganz herzlich gedankt!

Nun übernehmen wir die Aufgabe, uns für die Zeitschrift Grundwasser zu engagieren. Mit einem großartigen Team assoziierter Editoren und der Unterstützung von Ruth Kaufmann-Knoke, Patricia Schüll und Jörg Böger im Rücken freuen wir uns auf die neue Herausforderung.

Wir erhoffen uns vielfältige Beiträge, neue Aspekte, spannende Themenhefte und interessante Ideen. Wir sind überzeugt davon, dass das Thema Grundwasser sowohl im Hinblick auf die quantitativen als auch die qualitativen Aspekte in Zukunft noch an Bedeutung gewinnen wird. Deshalb ist auch die Zeitschrift Grundwasser als Ort für den fachlichen Austausch und die Diskussion wichtiger denn je. Wir werden uns bemühen, die Zeitschrift Grundwasser für Autoren und Leserschaft gleichermaßen attraktiv, anregend und aktuell zu gestalten.

Ihre

Gudrun Massmann und Traugott Scheytt

G. Massmann $(\bowtie)$

Carl von Ossietzky Universität Oldenburg,

Oldenburg, Deutschland

E-Mail: gudrun.massmann@uni-oldenburg.de

T. Scheytt

Technische Universität Berlin,

Berlin, Deutschland

E-Mail: traugott.scheytt@tu-berlin.de 